\title{
ON TRANSVERSALITY
}

\author{
by J. W. BRUCE
}

(Received 27th March 1985)

The notion of transversality has proved of immense value in differential topology. The Thom transversality lemma and its many variants show that transversality is a dense, and often open, property. In one parameter families the occurrence of non-transversality is inevitable; for example one cannot pull two linked curves in $\mathbb{R}^{3}$ apart without a non transverse intersection. The aim of this note is to prove the following. In any generic family of mappings each map in the family fails to satisfy some fixed transversality conditions at worst at isolated points, and even at these points in rather special sorts of way. So, returning to the above example, given two space curves $C_{1}$ and $C_{2}$ without a (necessarily non-transverse) intersection we expect, in any generic isotopy of $C_{2}$, that it will meet $C_{1}$, if at all, at isolated points. In particular generically we do not expect $C_{1}$ and $C_{2}$, any time, to have an arc in common

The key idea is the replacement of "transversality" by "weak transversality", and with it the replacement of the idea of "submersion" by "finite singularity type". Our main results then follow from well-established facts of singularity theory.

Some geometrical applications are given in Section 2 of the ideas and techniques employed in the proof of our general results given in Section 1. Unfortunately each geometrical application requires its own transversality result, and so no general theorem seems likely to cover a substantial number of applications. This point is well illustrated by a glance through [7]. Thus our definition of weak transversality in (1.4) and the method of proof of our main results $(1.1,1.3)$ can be regarded as prototypes in much the same way as the standard Thom transversality results $([2$, p. 68], $[1$, p. 54]) are for the usual notation of transversality.

1.

Let $A$ and $B$ be smooth $\left(C^{\infty}\right)$ manifolds, $C \subset B$ a smooth submanifold of $B$ and $f: A \rightarrow B$ a smooth map. Recall that $f$ is transverse to $C$ if and only if for each point $x \in A$ with $f(x) \in C$ we have

$$
T_{x} f\left(T_{x} A\right)+T_{f(x)} C=T_{f(x)} B
$$

where $T_{x} f$ is the tangent map of $f$ at $x, T_{x} A$ the tangent space to $A$ at $x$ etc.

Transversality is an extremely useful property (see [2], [7] for some nice geometrical applications of the idea). If $f$ is transverse to $C$, written $f \pi C$, then $f^{-1}(C)$ is a smooth 
submanifold of $A$ and its codimension coincides with that of $C$ in $B$. Hence the "solution set of the equation" $f(A) \subseteq C$ has the nicest possible local structure and is of the expected dimension. Moreover in the set of smooth mappings $f: A \rightarrow B$, denoted by $C^{\infty}(A, B)$, those transverse to $C$ are residual; when $C$ is closed they are in fact open and dense. (See [1] Chapter II for proofs of these results). Of course another attractive feature of the notion is that since it involves checking conditions on linear maps it is computationally quite reasonable.

Instead of a single map $f: A \rightarrow B$ we may consider a smooth map $F: A \times U \rightarrow B$, which we think of as a family of maps $F_{u}: A \rightarrow B$, one for each $u \in U$, where $F_{u}(x)=F(x, u)$. It will certainly no longer be true that by a small perturbation of $F$ we can ensure that $F_{u}: A \rightarrow B$ is transverse to $C$ for each $u \in U$, as mentioned in the introduction. Two connected questions come to mind. First how badly will the maps $F_{u}$ fail to be transverse to $C$ ? And secondly what will the sets $F_{u}^{-1}(C)$ look like?

Theorem 1.1. Let $A, B, C, U$ be as above. For a residual set of mappings $F \in C^{\infty}(A \times U, B)$ the following holds:

(a) for each $u \in U$ the map $F_{u}: A \rightarrow B$ is transverse to $C$ except possibly on a discrete set of points;

(b) for each $u \in U$ the set $F_{u}^{-1}(C)$ is smooth except possibly at a discrete set of points. At each of these exceptional points $F_{u}^{-1}(C)$ is locally diffeomorphic to the germ of an algebraic variety of the same codimension as that of $C$ in $B$, with the exceptional point corresponding to an isolated singular point of the variety.

Notes 1.2. (a) In particular $F_{u}^{-1}(C)$ is smooth except at isolated points where it has topologically a nice cone-like structure.

(b) As we shall see in the proof there is a natural local complexification of the above situation and in this case $F_{u}^{-1}(C)$, at its non smooth points, is an isolated singular point of a complete intersection and a great deal is known about such singularities (See [4]).

(c) The case when the codimension of $C$ in $B$, $\operatorname{cod}_{B} C$ satisfies $\operatorname{cod}_{B} C \geqq \operatorname{dim} A$ is of some interest. Here generically $F_{u}^{-1}(C)$ is a discrete set of points (or empty) for all $u \in U$. As a special case when $\operatorname{dim} A=\operatorname{dim} B$ and $C$ is a point this can be interpreted as "blowing-up phenomena are infinitely rare in differential topology".

The Theorem above can be extended in a number of ways. One natural candidate is to consider the case when $B$ is the total space of a $C^{\infty}$-bundle, so suppose that there is a submersion $\phi: B \rightarrow D$ for some smooth manifold $D$.

Theorem 1.3. Let $A, B, D, U$ and $\phi: B \rightarrow D$ be as above. Then for a residual set of mappings $F \in C^{\infty}(A \times U, B)$ the conclusions of Theorem 1.1 hold for all submanifolds $C=\phi^{-1}(d), d \in D$.

Notes 1.2 continued. (d) The particular case when $D=B$ and $\phi$ is the identity is worthy of note. When $\operatorname{dim} A=\operatorname{dim} B$ it follows that non locally finite maps between manifolds of the same dimension are infinitely rare. (Compare [3]).

The rest of this section will be taken up with the proofs of Theorems 1.1 and 1.3. 


\section{Start of Proof of Theorem 1.1.}

The basic idea is very simple. Let $f: A \rightarrow B \supset C$ be as above, with $x \in A, f(x) \in C$. If we write $C$ near $f(x)$ as the inverse image of a regular value of a smooth germ

$$
g:(B, f(x)) \rightarrow\left(\mathbb{R}^{m}, 0\right)
$$

then $f$ is transverse to $C$ at $x$ if and only if $g \circ f:(A, x) \rightarrow\left(\mathbb{R}^{m}, 0\right)$ is the germ of a submersion. In some sense Sard's theorem ([1] p. 34) leads one to the conclusion that $g \circ f$ usually will be a submersion; indeed Sard's theorem is a crucial ingredient in all proofs of the transversality result. Replacing $f$ by a family $F: A \times U \rightarrow B$ singularities at $x$ of $g \circ F_{u}$ are unavoidable. Instead of asking that $g \circ f$ is a submersion at $x$ we can merely ask that it is of finite singularity type (F.S.T.). (See [5], p. 205, [6], p. 513). Here are the crucial facts we shall need concerning this notion. First some notation. The ring of germs of smooth functions $f:\left(\mathbb{R}^{n}, 0\right) \rightarrow \mathbb{R}$ is denoted by $\mathscr{E}_{n}$; its maximal ideal of functions vanishing at 0 by $m_{n}$. The $\mathscr{E}_{n}$-module of germs $\left(\mathbb{R}^{n}, 0\right) \rightarrow \mathbb{R}^{p}$ is denoted by $\mathscr{E}(n, p)$, and any germ $f:\left(\mathbb{R}^{n}, 0\right) \rightarrow\left(\mathbb{R}^{p}, 0\right)$ induces a ring homomorphism $f^{*}: \mathscr{E}_{p} \rightarrow \mathscr{E}_{n}$ via composition.

I. A map germ $f:\left(\mathbb{R}^{n}, 0\right) \rightarrow\left(\mathbb{R}^{p}, 0\right)$ is of F.S.T. if and only if the $\mathscr{E}_{n}$-module

$$
\mathscr{E}_{n}\left\{\partial f / \partial x_{1}, \ldots, \partial f / \partial x_{n}\right\}+f^{*} m_{p} . \mathscr{E}(n, p) \subset \mathscr{E}(n, p)
$$

denoted by $T_{e} K . f$, contains $m_{n}^{k} \cdot \mathscr{E}(n, p)$ for some $k \geqq 1$. This may be taken as the definition of F.S.T. (Here the first term in the expression above is the $\mathscr{E}_{n}$-module generated by the $\partial f / \partial x_{i}$.)

II. If $f:\left(\mathbb{R}^{n}, 0\right) \rightarrow\left(\mathbb{R}^{p}, 0\right)$ is of F.S.T. then $f$ is contact equivalent ([5], p. 209, [6], p. 482) to its $r$-jet $j^{r} f$, for some $r<\infty$. (Indeed given the inclusion of I we can take $r=k$.) In particular the germs $\left(f^{-1}(0), 0\right)$ and $\left(\left(j^{r} f\right)^{-1}(0), 0\right)$ are locally diffeomorphic. Moreover if two germs $f_{1}, f_{2}:\left(\mathbb{R}^{n}, 0\right) \rightarrow\left(\mathbb{R}^{p}, 0\right)$ are contact equivalent and $f_{1}$ is of F.S.T. than $f_{2}$ is also.

III. If $f$ is as in II the polynomial $g=j^{r} f$ can be considered as a complex map germ $g:\left(\mathbb{C}^{n}, 0\right) \rightarrow\left(\mathbb{C}^{p}, 0\right)$. Then if $\Sigma g$ is the set of critical points of $g$ (i.e. points $x$ where $T_{x} g$ is not onto) this set meets $g^{-1}(0)$, locally, only at 0 .

IV. For any positive integer $k$ the set of polynomial germs $g:\left(\mathbb{R}^{n}, 0\right) \rightarrow\left(\mathbb{R}^{p}, 0\right)$ for which $T_{e} \mathbf{K} \cdot g+m_{n}^{k} \cdot \mathscr{E}(n, p)$ has codimension $\geqq k$ in $\mathscr{E}(n, p)$ is an algebraic subset $W_{k}$, of the vector space $J_{0}^{k}(n, p)$ of such germs, of codimension say $d_{k}$. Moreover $d_{k}$ is a non decreasing sequence, $\lim _{k \rightarrow \infty} d_{k}=\infty$, and if for any germ $f:\left(\mathbb{R}^{n}, 0\right) \rightarrow\left(\mathbb{R}^{p}, 0\right)$ we have $j^{k} f \notin W_{k}$ then $f$ is contact equivalent to $j^{k} f$ and both are of F.S.T. See [6, p. 513] for proofs of these results.

Result IV above can be roughly interpreted to mean that germs not of F.S.T. are infinitely rare.

We now make the obvious definition. 
Definition 1.4. Let $f: A \rightarrow B\lrcorner C$ be as above. Then $f$ is weakly transverse to $C$ if for each point $x \in A$ with $f(x) \in C$ the following holds. Writing $C$ locally as the inverse image of the regular value 0 of a germ $g:(B, f(x)) \rightarrow \mathbb{R}^{m}, 0$ the composite $g \circ f$ : $(A, x) \rightarrow\left(\mathbb{R}^{m}, 0\right)$ is of F.S.T.

Lemma 1.5. The definition above is independent of the choice of submersion $g$.

Proof. If $g_{1}$ and $g_{2}$ are two such germs the ideals $\left(g_{1} \circ f\right)^{*} m_{m},\left(g_{2} \circ f\right)^{*} m_{m}$ coincide, for they coincide with $f^{*} I$ where $I$ is the ideal of germs $h:(B, x) \rightarrow(\mathbb{R}, 0)$ which vanish on $C$. It now follows from [5, p. 212] that $g_{1} \circ f$ and $g_{2} \circ f$ are contact equivalent, and so $g_{1} \circ f$ is of F.S.T. if and only if $g_{2} \circ f$ is.

We shall actually show that for a residual set of mappings $F$ in $C^{\infty}(A \times U, B)$ the maps $F_{u}: A \rightarrow B$ are weakly transverse to $C$ for all $u$ in $U$; the proof of Theorem 1.1 then easily follows. For 1.1(a) is a consequence of Property III above of germs of F.S.T.: if $F_{u}$ is weakly transverse to $C$ at some point $x$ then either $F$ is transverse to $C$ at $x$ or fails to be transverse locally only at $x$. The proof of $1.1(b)$ on the other hand is a direct consequence of Property II which states that germs of F.S.T. are finitely determined.

Given $A, B, C$ as above we shall now construct a subset $\Omega_{k}(C)$ of each jet space $J^{k}(A, B)$ with the property that $f: A \rightarrow B$ is weakly transverse to $C$ if and only if $j^{k} f: A \rightarrow J^{k}(A, B)$ misses $\Omega_{k}(C)$. We first construct $\Omega_{k}$ locally. So let $A=\left(\mathbb{R}^{n}, 0\right), B=\left(\mathbb{R}^{p}, 0\right)$ and $C=\left(\mathbb{R}^{q}, 0\right) \subset\left(\mathbb{R}^{p}, 0\right)$ be given the vanishing of the last $p-q$ co-ordinates of $\mathbb{R}^{p}$. We denote by $J_{0}^{k}(n, p)$ the space of polynomial germs $\left(\mathbb{R}^{n}, 0\right) \rightarrow\left(\mathbb{R}^{p}, 0\right)$ of degree $\leqq k$. Such a germ $g$ will be weakly transverse to $\left(\mathbb{R}^{q}, 0\right)$ at 0 if and only if $\Pi \circ g:\left(\mathbb{R}^{n}, 0\right) \rightarrow\left(\mathbb{R}^{p-q}, 0\right)$ is of F.S.T. where $\Pi:\left(\mathbb{R}^{p}, 0\right) \rightarrow\left(\mathbb{R}^{p-q}, 0\right)$ is projection to the last $p-q$ co-ordinates of $\mathbb{R}^{p}$. Composition with $\Pi$ induces a linear surjection $\Pi_{*}: J_{0}^{k}(n, p) \rightarrow J_{0}^{k}(n, p-q)$. If $W_{k} \subset J_{0}^{k}(n, p-q)$ is the set of polynomial germs of IV above we set $\Omega_{k}^{l}=\Pi_{*}^{-1}\left(W_{k}\right)$. Clearly $\Omega_{k}^{l}$ is also a union of algebraic varieties of the same codimension in $J_{0}^{k}(n, p)$ as that of $W_{k}$ in $J_{0}^{k}(n, p-q)$. The lemma above shows that $\Omega_{k}^{l}$ is independent of the choice of projection $\Pi$. Moreover if $\phi_{1}:\left(\mathbb{R}^{n}, 0\right) \rightarrow\left(\mathbb{R}^{n}, 0\right)$ is the germ of a diffeomorphism, as is $\phi_{2}:\left(\mathbb{R}^{p}, 0\right) \rightarrow\left(\mathbb{R}^{p}, 0\right)$ with $\phi_{2}$ preserving $\left(\mathbb{R}^{p-q}, 0\right) \subset\left(\mathbb{R}^{p}, 0\right)$ it is not difficult to see that for any germ $g:\left(\mathbb{R}^{n}, 0\right) \rightarrow\left(\mathbb{R}^{p}, 0\right)$ we have $j^{k} g \in \Omega_{k}^{l}$ if and only if $j^{k}\left(\phi_{2} \circ g \circ \phi_{1}\right) \in \Omega_{k}^{l}$. For we claim that $\Pi \circ \phi_{2} \circ g \circ \phi_{1}$ and $\Pi \circ g$ are contact equivalent; it is clearly enough to show that $\Pi \circ g$ and $\Pi \circ \phi_{2} \circ g$ are, but this follows from Lemma 1.5 , for $\Pi$ and $\Pi \circ \phi_{2}$ are two choices of suitable submersions for defining $\left(\mathbb{R}^{q}, 0\right) \subset\left(\mathbb{R}^{p}, 0\right)$.

We can now define the subset $\Omega_{k}(C) \subset J^{k}(A, B)$. We define it to be the set of $k$-jets $\sigma$ with target $\sigma \in C$ such that for any representative germ $f:(A, \bar{x}) \rightarrow(B, f(\bar{x}) \supset(C, f(\bar{x}))$ and any co-ordinate representation of $f, \tilde{f}:\left(\mathbb{R}^{n}, 0\right) \rightarrow\left(\mathbb{R}^{p}, 0\right) \supset\left(\mathbb{R}^{q}, 0\right)$ we have $j^{k} \tilde{f} \in \Omega_{k}^{l} \subset J_{0}^{k}(n, p)$. It follows from the above that $\Omega_{k}(C)$ is well defined. It is not difficult to see that since $\Omega_{k}^{l}$ can be given a manifold partition into manifolds of codimension $\geqq d_{k}$ the set $\Omega_{k}(C) \subset J^{k}(A, B)$ can be partitioned into manifolds of codimension $\geqq d_{k}+\operatorname{cod}_{B} C$, since locally $\Omega_{k}(C)$ is a product $A_{1} \times C_{1} \times \Omega_{k}^{l}$ where $A_{1}$ (resp. $C_{1}$ ) is an open set in $A$ (resp. $C$ ). Theorem 1.1 now follows from the Thom transversality lemma. For choosing $k$ sufficiently large we can ensure that $\Omega_{k}(C)$ is of codimension $>\operatorname{dim}(A \times U)$, and for a residual set of mappings $F \in C^{\infty}(A \times U, B)$ the jet extension $j_{1}^{k} F: A \times U \rightarrow J^{k}(A, B)$ defined by $j_{1}^{k} F(x, u)=j^{k} F_{u}(x)$ is transverse to the strata of $\Omega_{k}(C)$. Consequently $j_{1}^{k} F(A \times U)$ misses $\Omega_{k}(C)$ and for each $u \in U$ we have $F_{u}: A \rightarrow B$ weakly transverse to $C$ as required. 
Since a minor variant of the usual Thom transversality lemma of [1, p. 54], (7, p. 739] is required, because of the presence of the $U$-parameter space, we sketch the corresponding (slight) changes in the proof.

Lemma 1.6. Let $\Omega$ be a submanifold of the jet space $J^{k}(A, B)$. There is a residual set of smooth mappings $F \in C^{\infty}(A \times U, B)$ such that the jet extension

$$
j_{1}^{k} F: A \times U \rightarrow J^{k}(A, B)
$$

is transverse to $\Omega$.

Proof. First recall the fundamental fact behind all transversality proofs ([1, p. 54], [7, p. 739]). If $G: N \times U \rightarrow P$ is a submersion, $Q \subset P$ a submanifold then the restriction $g_{u}=F \mid N \times\{u\}: N \times\{u\} \rightarrow P$ is transverse to $Q$ for almost all $u \in U$, in the sense of Lebesgue measure. This proves density of transverse maps, the residual property follows by standard arguments. The key to proving the transversality result we require, then, is to find for any $F \in C^{\infty}(A \times U, B)$ a family $G: A \times U \times V \rightarrow B$ with $0 \in V \subset \mathbb{R}^{n}$, such that $G(a, u, 0)=F(a, u)$ for all $(a, u) \in A \times U$ and $j_{1}^{k} G: A \times U \times V \rightarrow J^{k}(A, B)$ is a submersion. However, in $[7, p .740]$ Wall describes a method for obtaining a family $G$ as above with the jet extension $j^{k} G: A \times U \times V \rightarrow J^{k}(A \times U, B)$ a submersion. Now there is a canonical projection $\Pi: J^{k}(A \times U, B) \rightarrow J^{k}(A, B)$ defined by $\Pi\left((a, u), f(a, u), j^{k} f(a, u)\right)=(a, f(a, u)$, $\left.j^{k} f_{u}(a)\right)$ which is also a submersion, and since $\Pi \circ j^{k} G=j_{1}^{k} G$ the result follows. The proof of this lemma concludes the proof of Theorem 1.1.

Proof of Theorem 1.3. Clearly we want to show that for a residual set of mappings $F \in C^{\infty}(A \times U, B)$ we have $F_{u}: A \rightarrow B$ weakly transverse to $\phi^{-1}(d)$ for each $d \in D$. In other words we want $\phi \circ F_{u}: A \rightarrow D$ of F.S.T. for each $u \in U$. We have, by IV above, for each $k$ a subset $\Omega_{k} \subset J^{k}(A, D)$ of jets with the property that if $f: A \rightarrow D$ satisfies $j^{k} f(A) \cap \Omega_{k}=\varnothing$ then $f$ is of F.S.T. Moreover the codimension of $\Omega_{k}$, which is locally algebraic, tends to infinity as $k$ increases. Choose $k$ so that this codimension exceeds $\operatorname{dim}(A \times U)$. We need only show that for a residual set of $F \in C^{\infty}(A \times U, B)$ the jet extension

$$
j_{1}^{k}(\phi \circ F): A \times U \rightarrow J^{k}(A, D)
$$

is transverse to $\Omega_{k}$. Thus we are reduced to proving a variant of the usual Thom transversality lemma. However composition with $\phi$ induces a map $\phi_{*}: J^{k}(A, B) \rightarrow J^{k}(A, D)$ which is easily checked to be a submersion. Again it is not now difficult to see that the usual proof of the transversality lemma, as in [7] for example, can be adapted as above to give this slightly stronger version here.

\section{Geometrical applications}

In this section we briefly mention some geometrical applications of the ideas rather than the results discussed in Section 1. Applications of the fact that mappings between smooth equidimensional manifolds are in general finite are given in [3] in some detail. 
In what follows the techniques employed in [3] can be easily adapted to fill in gaps in the proofs. As remarked above each geometrical application requires its own transversality result.

Application 2.1. Let $M$ and $N$ be smooth submanifolds of $\mathbb{R}^{n}$. For almost any such $M$ and any $N$ the intersection of $M$ with any translate $N+\{a\}$ of $N$, for $a \in \mathbb{R}^{n}$, is smooth in $M$ (of the correct codimension) except at a discrete set of points as in Theorem 1.1(b) where the intersection is locally diffeomorphic to a real algebraic variety with an isolated singular point. In fact the inclusion $M \rightarrow \mathbb{R}^{n}$ is weakly transverse to $N+\{a\}$ for all $a \in \mathbb{R}^{n}$.

Proof. We shall suppose that $N$ is the inverse image of the regular value 0 by some smooth map $g: \mathbb{R}^{n} \rightarrow \mathbb{R}^{m}$. (The general case is a straightforward if tedious deduction using the fact that locally $N$ can be written as such an inverse image.) We have a smooth map $G: M \times \mathbb{R}^{n} \rightarrow \mathbb{R}^{m}$ defined by $G(x, a)=g(i(x)+a)$ where $i: M \rightarrow \mathbb{R}^{n}$ is the embedding of $M$ in $\mathbb{R}^{n}$. The result now follows if we can show that for almost any embedding $i$ the maps $G_{a}: M \rightarrow \mathbb{R}^{m}$, for $a \in \mathbb{R}^{n}$, are of F.S.T. (Compare the remarks following Lemma 1.5.) Using the fact that $g$ is a submersion it is easy to show that for a residual set of embeddings $i: M \rightarrow \mathbb{R}^{n}$ the jet extension $j_{1}^{k} G: M \times \mathbb{R}^{n} \rightarrow J^{k}\left(M, \mathbb{R}^{m}\right)$ will be transverse to the sets $\Omega_{k} \subset J^{k}\left(M, \mathbb{R}^{m}\right)$, using for example the ideas in [7].

The proof is as follows. We take $P^{r}$ to be the space of polynomial maps $\mathbb{R}^{n} \rightarrow \mathbb{R}^{n}$ of degree $\leqq r$ and let $U$ be a neighbourhood of the identity map Id in $P^{r}$ with the property that for any $f \in U$ the restriction of $f$ to $M$ is an embedding. We then consider $\widetilde{G}: M \times \mathbb{R}^{n} \times U \rightarrow \mathbb{R}^{m}$ defined by

$$
\tilde{G}(x, a, f)=g(f \circ i(x)+a) .
$$

Clearly $\tilde{G}(x, a, \mathrm{Id})=G(x, a)$; we now need to show that the jet-extension

$$
j_{1}^{k} \tilde{G}: M \times \mathbb{R}^{n} \times U \rightarrow J^{k}\left(M, \mathbb{R}^{n}\right)
$$

is a submersion, if $r$ is sufficiently large, at $M \times \mathbb{R}^{n} \times\{\mathrm{Id}\}$.

Let $F(x, a, f)=f \circ i(x)+a$, with jet extension

$$
j_{1}^{k} F: M \times \mathbb{R}^{n} \times U \rightarrow J^{k}\left(M, \mathbb{R}^{n}\right) .
$$

We claim it is enough to prove that this is a submersion at $M \times \mathbb{R}^{n} \times\{I d\}$; for the natural map $g^{*}: J^{k}\left(M, \mathbb{R}^{n}\right) \rightarrow J^{k}\left(M, \mathbb{R}^{m}\right)$, obtained by composition with $g$ is a submersion, because $g$ is, and $j_{1}^{k} \widetilde{G}=g^{*} \circ j_{1}^{k} F$. If $h$ is any polynomial map of degree $\leqq r$ as above, $h: \mathbb{R}^{n} \rightarrow \mathbb{R}^{n}$, then Id $+s h \in U$ for $s$ small, and

$$
\lim _{s \rightarrow 0} \frac{(\operatorname{Id}+s h) \circ i(x)-i(x)}{s}=h \circ i(x) .
$$

Since $i$ is an immersion clearly if $r \geqq k$ the map $j_{1}^{k} F$ is a submersion and the transversality result follows. 
The result required now follows by taking $k$ sufficiently large, for then a generic $G$ has a jet-extension $j_{1}^{k} G$ missing $\Omega_{k}$.

In fact a much stronger result is true. Namely if $U$ is any manifold (assumed, for simplicity, to be compact) and $M$ is compact and $\mathscr{J}: M \times U \rightarrow \mathbb{R}^{n}$ is a family of embeddings of $M$ then for a residual set of such $\mathscr{J}$ the above result holds for each $\mathscr{J}_{u}(M), u \in U$.

Application 2.2. Let $\xi$ be a smooth vector bundle with total space $E$, base space $B$ and let $U$ be a smooth manifold. Let $\Gamma^{\infty}(\xi ; U)$ be the space of smooth families of sections of $\xi$ parametrised by $U$. For a residual set of such sections $\sigma: B \times U \rightarrow E$ the restrictions $\sigma_{u}=\sigma \mid B \times\{u\}$ are weakly transverse to the zero section $E_{0}$ of $E$ for all $u$.

(Note that when the dimension of the fibres of $\xi$ coincides with the dimension of the base this implies that the $\sigma_{u}$ all have isolated zeros.)

Proof. Let $J^{k}(\xi)$ be the set of $k$-jets of sections $\sigma: B \rightarrow E$ of the bundle $\xi$. Locally $\xi$ is, of course, trivial so in turn $J^{k}(\xi)$ locally reduces to $J^{k}\left(B, \mathbb{R}^{n}\right)$, where $n$ is the dimension of the fibre of $\xi$. In each jet space $J^{k}(\xi)$ we have a set $\Omega_{k}$ of jets with the property that if $j^{k} \sigma: B \rightarrow J^{k}(\xi)$ avoids $\Omega_{k}$ the section $\sigma$ is weakly transverse to the zero section $E_{0}$ of $\xi$. We need only show that for a residual set $\sigma \in \Gamma^{\infty}(\xi, U)$ the jet map $j_{1}^{k} \sigma: B \times U \rightarrow J^{k}(\xi)$ is transverse to $\Omega_{k}$. But for some bundle $\eta$ the direct sum $\xi \oplus \eta$ is trivial, i.e. isomorphic to a bundle with total space $B \times \mathbb{R}^{N}$ (for some $N$ ) and has projection to the first factor $B$ as the bundle projection. Moreover there is a canonical bundle projection $P: \xi \oplus \eta \rightarrow \xi$. Given any family of sections $\sigma$ of $\xi \oplus \eta$ let

$$
\Phi_{1}: B \times U \times J^{k}\left(B, \mathbb{R}^{N}\right) \rightarrow \mathbb{R}^{N}
$$

be the map $\phi(x, u, \tau)=\sigma(x, u)+\tau(x)$. Clearly the jet extension $j_{l}^{k} \Phi_{1}: B \times U \times$ $J^{k}\left(B, \mathbb{R}^{N}\right) \rightarrow J^{k}\left(B, \mathbb{R}^{N}\right)$ is a submersion. So if

$$
\Phi: B \times U \times J^{k}\left(B, \mathbb{R}^{N}\right) \rightarrow B \times \mathbb{R}^{N}
$$

is the map $\Phi(x, u, \tau)=(x, \Phi(x, u, \tau))$ then $\Phi$ is a family of sections of $\xi$ and the jet extension $j_{1}^{k} \Phi: B \times U \times J^{k}\left(B, \mathbb{R}^{N}\right) \rightarrow J^{k}(\xi \oplus \eta)$ is a submersion.

Now if $\sigma$ is a family of sections of $\xi$ it can also be regarded as a family of sections of $\xi \oplus \eta$ as above. The map $P: \xi \oplus \eta \rightarrow \xi$ yields a submersion $P_{*}: J^{k}(\xi \oplus \eta) \rightarrow J^{k}(\xi)$ and hence $P_{*} \circ j_{1}^{k} \Phi$ is a submersion. Moreover $P_{*} \circ j_{1}^{k} \Phi=j_{1}^{k}(P \circ \Phi)$ and $P \circ \Phi$ is a family of sections of $\xi$ with $P \circ \Phi(x, u, 0)=\sigma_{u}(x)$ as required. Hence as usual for a residual set of smooth families of sections $\sigma$ the jet extension $j_{1}^{k} \sigma: B \times U \rightarrow J^{k}(\xi)$ is transverse to $\Omega_{k}$ and hence misses it for large enough $k$.

Application 2.3. Let $B$ have a foliation (see [3]) and let $\operatorname{Emb}^{\infty}(A, B)$ denote the space of smooth embeddings $A \rightarrow B$. Then for a residual subset of $\operatorname{Emb}^{\infty}(A, B)$ the embedded $A$ meets each leaf of the foliation transversally except at a discrete set of points, in the 
topology of the leaf. At these points the intersection is, in the leaf, of the type described in Theorem 1.1(b).

(The proof uses the ideas of Section 1 and [3] p. 149 and will not be given.)

Remarks 2.4. It would be nice to extend our main results to deal with jet extensions, and not merely smooth mappings. So for example in an arbitrarily large family of mappings $F: A \times U \rightarrow B$ between 2-manifolds one would expect the singular set of each $F_{u}$ to be a curve with isolated singularities (diffeomorphic to those of a real algebraic curve) and, moreover, one expects the differential of $F_{u}$ here to have rank $\geqq 1$ and $F_{u}$ to be a fold along this curve except, at worst, at isolated points. These results would certainly follow if for each $u$ the jet extension $j^{2} F_{u}: A \rightarrow J^{2}(A, B)$ was weakly transverse to the natural stratification of $J^{2}(A, B)$. (Some of these results follow fairly easily if each $F_{\mu}$ is merely of F.S.T. but certainly not all of them; the map $(x, y) \mapsto\left(x, y^{3}\right)$ is of F.S.T. but does not have the properties above.)

The problem with proving such theorems lies with the corresponding transversality results and is related to the inbuilt symmetry in the jet spaces $J^{k}\left(A, J^{l}(A, B)\right)$ one would have to consider. The same difficulty arises in the following situation.

Given a generic surface $M$ in $\mathbb{R}^{3}$ the set of points where the Gaussian curvature vanishes, the parabolic set, is a smooth curve. Along this curve the normal height functions have a degenerate singularity of type $A_{2}$ or, at points, of type $A_{3}$ (see [7] for the notation). Again one expects in arbitrarily large generic families of such surfaces the parabolic set to be a curve and at all but isolated points the normal height function to be of type $A_{2}$. In particular one expects these height functions to be of corank 2 only at isolated points. Writing a surface $M$ locally, at $0 \in \mathbb{R}^{3}$, as $z=f(x, y)$ with $(\partial f / \partial x)=$ $(\partial f / \partial y)=0$ at 0 this corank 2 condition is that $\left(\partial^{2} f / \partial x^{2}\right)=\left(\partial^{2} f / \partial x \partial y\right)=\left(\partial^{2} f / \partial y^{2}\right)=0$ also at 0 . The inbuilt symmetry here is partly reflected in the fact that the higher derivatives of these three expressions are clearly not independent. It is, of course, the existence of such symmetries which provides the only possible obstruction to proving the required transversality result.

\section{REFERENCES}

1. M. Golubitsky and V. Guillemin, Stable mappings and their singularities, Graduate Texts in Mathematics 14 (Springer-Verlag, 1973).

2. V. Guillemin and A. Pollack, Differential Topology (Prentice Hall, 1974).

3. H. Kurland and J. Robin, Infinite Codimension and Transversality, Dynamical Systems, Warwick, Lecture Notes in Mathematics 468 (Springer 1974).

4. E. J. N. LoolJenga, Isolated Singular Points on Complete Intersections, London Math. Soc. Lecture Note Series 77 (Cambridge Univ. Press, 1984).

5. J. Martinet, Singularities of Smooth Functions and Maps, London Math. Soc. Lecture Note Series 58 (Cambridge Univ. Press, 1982). 
6. C. T. C. WALl, Finite determinancy of smooth mappings, Bull. London Math. Soc. 13 (1981), 481-539.

7. C. T. C. WALL, Geometric Properties of Generic Differentiable Manifolds in Geometry and Topology, Vol. III, Lecture Notes in Maths. 597 (Springer, 1976).

School of Mathematics

UNIVERSITY OF NewCASTLE UPON TYNE

NewCastle UPON TYNe NE1 7RU 